\title{
A LOW-ENERGY RING LATTICE DESIGN FOR THE PEP-N PROJECT *
}

\author{
M. E. Biagini ${ }^{\dagger}$, Y. Cai, J. Seeman, M. Sullivan, U. Wienands, SLAC, Stanford, CA 94025 \\ M. Placidi, CERN, CH-1211 Geneva 23, Switzerland
}

\begin{abstract}
The PEP-N project at SLAC [1] consists of a Very Low Energy small electron Ring (VLER) that will collide with the low-energy $3.1 \mathrm{GeV}$ positron beam (LER) of PEP-II, producing center-of-mass energies between $1.1 \mathrm{GeV}$ and the $\mathrm{J} / \psi$. The beams will collide head-on and will be separated in the detector magnetic field which is part of the Interaction Region (IR) [2]. The IP $\beta$ functions were chosen such as to optimize both luminosity and beambeam tune shifts, while keeping the LER tune shifts small. This paper describes the lattice of the VLER for the 'baseline' design at $500 \mathrm{MeV}$.
\end{abstract}

\section{INTRODUCTION}

A proposal to build a new low-energy electron storage ring that will collide with the existing $3.1 \mathrm{LER}^{+}$beam in the IR12 straight section of PEP-II was made in September 2000 [3]. The energy range of the electron ring will be 100 to $800 \mathrm{MeV}$ resulting in a center-of-mass energy ranging from 1.1 to $3.1 \mathrm{GeV}$. The expected luminosity at $500 \mathrm{MeV}$ is about $10^{31} \mathrm{~cm}^{-2} \mathrm{~s}^{-1}$. PEP-N is to be operated simultaneously with the PEP-II collider and is designed to not interfere with the peak luminosity operation of PEP-II for BaBar data collection. The physics case [4] is mainly focused on the measurement of $\mathrm{R}$, the ratio between multi-hadron and muon pair cross sections, in an energy range were data are lacking or have large errors. These measurements will also allow to reduce the error on the calculated muon magnetic momentum $g_{\mu}-2$. The asymmetry of the beam energies will produce a favorable particle boost for measuring the poorly measured and understood nucleon form factors in the time-like region.

\section{MACHINE LAYOUT}

The VLER storage ring has been designed in order to meet the following requirements:

- to operate with an energy variable between 100 and $800 \mathrm{MeV}$;

- to provide the flexibility to keep the beam emittance constant while varying the energy;

- to collide with LER without perturbing the BaBar operation;

- to fit in the Hall 12 (20 m x $7 \mathrm{~m})$, leaving enough space for the injection line from the Linac;

- to allow for head-on collisions with a minimum impact on the detector;

All these requirements are fulfilled by the present

*Work supported by the U.S. Department of Energy, contract DEAC03-76SF00515.

†Also LNF-INFN, Italy, biagini@slac.stanford.edu baseline design at $500 \mathrm{MeV}$. The ring circumference was chosen to be a multiple of the LER bunch spacing. However, for lack of space, we could not choose a VLER length that is a sub-multiple of the LER length. As a consequence the $\mathrm{e}^{-}$bunches will collide with many different $\mathrm{e}^{+}$bunches. Possible implications of this choice will be discussed later.

\section{LATTICE DESIGN}

The ring has a two-fold symmetry with a circumference of $45.36 \mathrm{~m}$. This is 36 times the LER by 2 bunch spacing. The beams collide head-on and are brought into and out of collision by the detector magnetic field. The main lattice parameters are summarized in Table 1. A RF voltage of $100 \mathrm{kV}$ was assumed. The emittance is the uncoupled one. Dipole fields and quadrupole gradients refer to the maximum energy of $800 \mathrm{MeV}$.

Table 1: Lattice Parameters

\begin{tabular}{|c|c|c|c|}
\hline E $(\mathrm{GeV})$ & $0.1-0.8$ & C (m) & 45.36 \\
\hline I (mA) & $10-140$ & $\begin{array}{c}\mathrm{L}\left(\mathrm{cm}^{-2} \mathrm{~s}^{-}\right. \\
\left.{ }^{1}\right) \times 10^{31}\end{array}$ & $2-200$ \\
\hline B field $(T)$ & 1.63 & Max B' (T/m) & 10. \\
\hline frev (MHz) & 6.6 & $\mathbf{h}$ & 72 \\
\hline$\beta_{\mathrm{x}} *(\mathrm{~cm})$ & 30. & $\beta_{\mathrm{y}}{ }^{*}(\mathrm{~cm})$ & 2.85 \\
\hline $\operatorname{INT}\left(v_{x}\right)$ & 3. & INT $\left(v_{y}\right)$ & 3. \\
\hline $\operatorname{Max} \operatorname{IR} \beta_{x}(m)$ & $60 .-80$. & $\operatorname{Max} \operatorname{IR} \beta_{\mathrm{y}}(\mathrm{m})$ & 100. \\
\hline$\eta_{\mathrm{x}}{ }^{*}(\mathrm{~m})$ & 0 . & $\operatorname{Max} \eta_{x}(m)$ & $2.2-3.5$ \\
\hline$\sigma_{x}{ }^{*}(\mu)$ & 274. & $\sigma_{y}^{*}(\mu)$ & 84. \\
\hline$\varepsilon_{0}(\mathrm{~nm})$ & 500. & $\kappa=\varepsilon_{\mathrm{y}} / \varepsilon_{\mathrm{x}}$ & 1 \\
\hline$\alpha_{c}$ & .052 & $\sigma_{1}(\mathrm{~cm})$ & $1.2-0.7$ \\
\hline$\sigma_{\mathrm{E}} / \mathrm{Ex}_{10}{ }^{+04}$ & 3.6 & $\mathrm{U}_{0}(\mathrm{KeV} /$ turn $)$ & $.02 .-22$. \\
\hline$\tau_{\mathrm{x}}(\mathrm{ms})$ & $1000-5$ & $\mathbf{v}_{\mathrm{s}}$ & .011 \\
\hline
\end{tabular}

The ring layout is sketched in Fig. 1. The dimensions are the actual hall size $(20 \mathrm{~m} \times 7.16 \mathrm{~m})$, the yellow circle is the detector field.

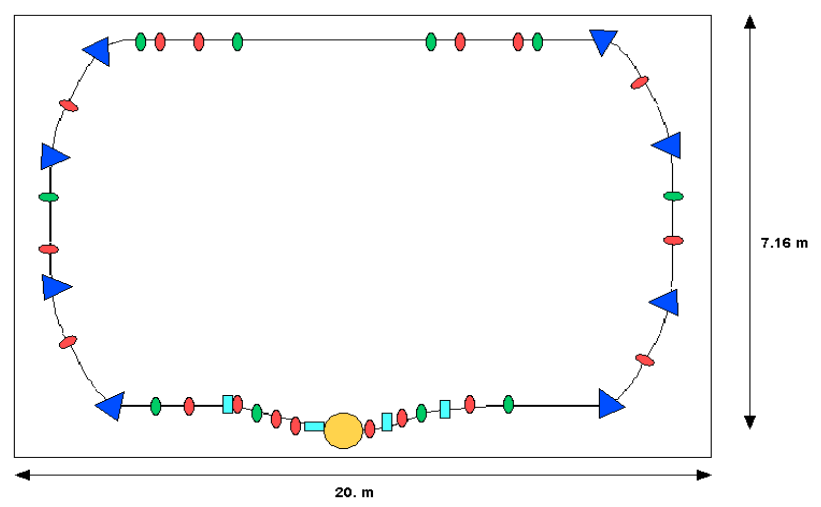

Figure 1: Ring layout. 
The ring lattice consists of two straight sections and two arcs. In the IR the detector is shifted downstream the collision point by $25 \mathrm{~cm}$ in order to increase the detector coverage for boosted particles. The focusing at the IP and along the IR is provided by two QD-QF doublets. The first quadrupole in the doublet is a permanent magnet on the downstream side. This design maximizes the detector solid angle. The first quadrupole on the upstream side is a shared magnet that helps to separate the beams. The IP beta functions were chosen in order to optimize both luminosity and beam-beam tune shifts: $\beta_{\mathrm{x}}{ }^{*}=30 \mathrm{~cm}, \beta_{\mathrm{y}}{ }^{*}$ $=2.85 \mathrm{~cm}$. A detailed description of the IR can be found in Ref. [2].

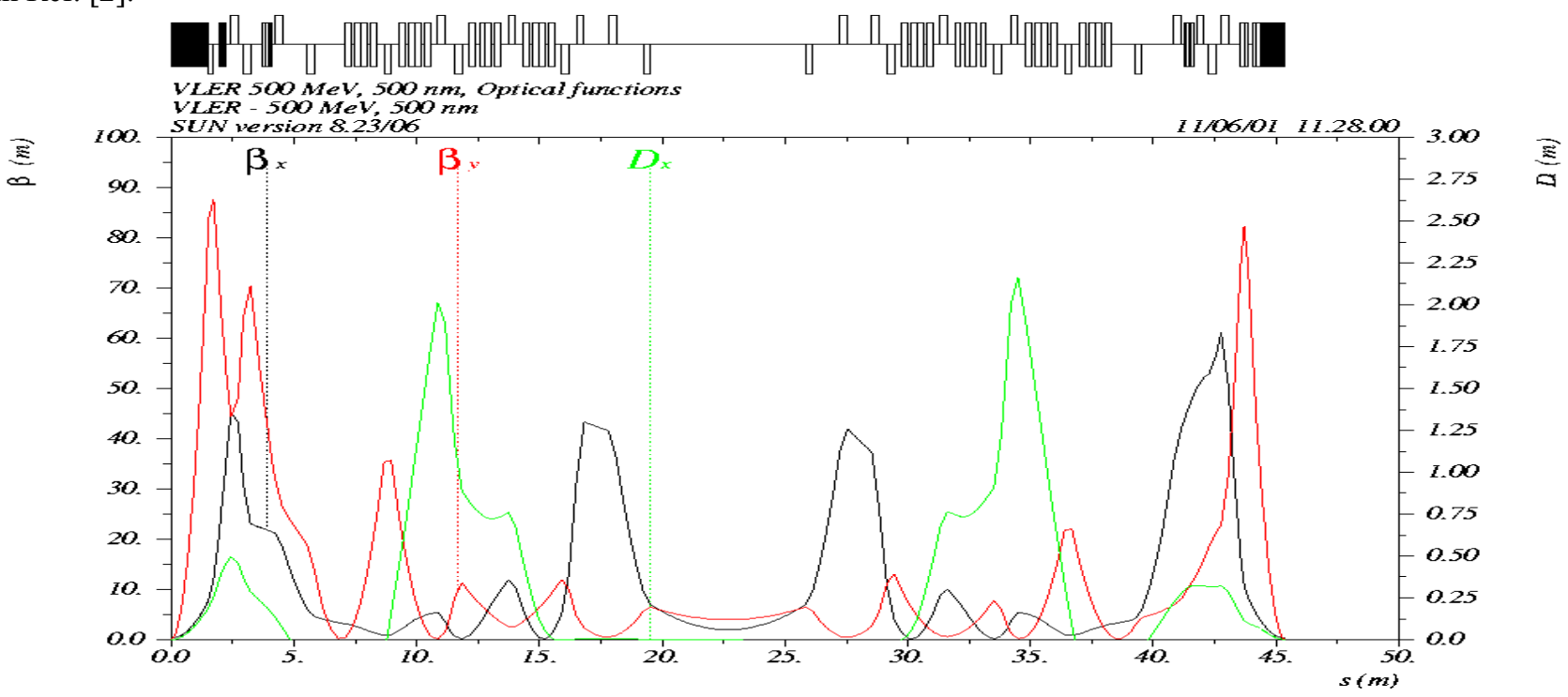

Figure 2: Optical functions for the $500 \mathrm{MeV}$ lattice $\left(\beta_{\mathrm{x}}\right.$ black, $\beta_{\mathrm{y}}$ red, $\eta_{\mathrm{x}}$ green).
The other straight will house the injection kickers, septum, feedbacks and one RF cavity. In the injection straight there are four QD-QF doublets, which will be used for tune adjustments and for maintaining nearly symmetric optical functions between the two arcs. The horizontal dispersion vanishes at the IP as well as in the $\mathrm{RF} /$ injection region.

Due to the limited available space, a FODO cell solution as in the LER and HER arcs of PEP-II was not possible. A compact arc design has been chosen which allows for both emittance control with energy and dispersion suppression in the RF and injection straights.
Each arc houses four dipoles, interleaved with 4 quadrupoles. The philosophy is to construct eight $1.28 \mathrm{~m}$ long dipoles with a peak field of $1.635 \mathrm{~T}$ at $800 \mathrm{MeV}$. Each dipole is segmented into sixteen, $0.08 \mathrm{~m}$ long steel pieces, to allow the magnet length to be shortened to increase the field at lower energies. The long magnet coils will not change even though some of the segments are removed. The segments will be removed when going to low energies, in order to keep four symmetrical submagnets. This will maintain the curvature of the beam trajectory, therefore the vacuum chamber will not need to be changed. This design increases synchrotron radiation at low energies, shortening the damping times, and allowing for higher beam-beam tune shifts with corresponding higher luminosity. The possibility of installing a $7.5 \mathrm{~T}, 1$ $\mathrm{m}$ long super-conducting wiggler in the $\mathrm{RF} /$ injection straight is also being studied.

The total number of normal conducting quadrupoles in the ring is 24 . They will be individually powered, to allow maximum lattice flexibility. Some extra space is available for closed orbit correctors, emittance-coupling skew quadrupoles and sextupoles.

Optical functions are presented in Fig. 2 for the $500 \mathrm{~nm}$, $500 \mathrm{MeV}$ lattice.

\section{BEAM DYNAMICS}

\subsection{Dynamic Aperture}

Due to the high natural chromaticity and the limited space available for sextupoles the optimization of the dynamic aperture has to be carefully studied. Four families of sextupoles are foreseen: three for chromaticity correction in the arcs, where the betatron functions in the two planes are quite well separated and the dispersion is maximum, and one in the injection straight to correct the beam tune shift with the particle amplitude. Due to space issues the phase shift between sextupoles will probably not be optimized to exactly cancel chromatic and geometric aberrations. The dynamic aperture with a fast tracking program was computed [3] for a previous version of the $500 \mathrm{MeV}$ lattice, with a lower emittance. Particles with initial conditions confined in a region of $\left( \pm 10 \sigma_{\mathrm{x}}{ }^{*}\right.$, $\left.+10 \sigma_{\mathrm{y}}{ }^{*}\right)$ at nominal coupling, and for three fixed energy deviations, corresponding to $\Delta \mathrm{p} / \mathrm{p}=\left(\begin{array}{lll}-10 & \sigma_{\mathrm{E}} / \mathrm{E}, 0,+10\end{array}\right.$ $\left.\sigma_{\mathrm{E}} / \mathrm{E}\right)$, were tracked for $3 \times 10^{5}$ turns, corresponding to one transverse damping time. Magnet errors and synchrotron oscillations have not been studied. The results are promising since the stable area was larger than $\pm 10 \sigma_{\mathrm{x}}$. 


\subsection{Beam Lifetime}

In an electron storage ring, the multiple Coulomb scattering of the charged electrons within a bunch leads to the growth of emittance in all three dimensions. The growth rates are proportional to the fourth power of the inverse of beam energy [5]. Hence, at the lower end of the energy range $(100 \mathrm{MeV})$, this effect could become the dominant factor in determining the equilibrium beam size.

To estimate the effect of the intrabeam scattering, the growth rates of emittance in all three dimensions as a function of energy were computed using MAD [6]. The lowest energy design lattice was used in the calculation, as it is the more problematic case for this effect. At all energies, the bunch length was fixed at $1 \mathrm{~cm}$, the horizontal emittance was kept at the constant value of 250 $\mathrm{nm}$, and the vertical emittance was assumed $10 \%$ of the horizontal one. The values of the charge per bunch at different energies were interpolated based on the design values. The growth rate turns out to be small compared to the damping rate due to the synchrotron radiation when the energy is larger than $200 \mathrm{MeV}$, and it increases rapidly once the energy drops below $200 \mathrm{MeV}$. This result indicates that below $200 \mathrm{MeV}$ it might be an issue to maintain a reasonable beam size and beam lifetime since there is no equilibrium distribution if the growth rate is larger the damping rate. However the larger vertical emittance $(50 \%$ of the horizontal in the present design), and smaller damping times can help in decreasing the growth rate.

The lifetime of the electron beam due to the Touschek effect was estimated as a function of the VLER energy using the simple formula by Le Duff [7] for flat beams. At all energies, the momentum acceptance is calculated with a fixed RF voltage of $100 \mathrm{kV}$. The results of calculation are shown in Figure 3 as a function of the number of particles per bunch.

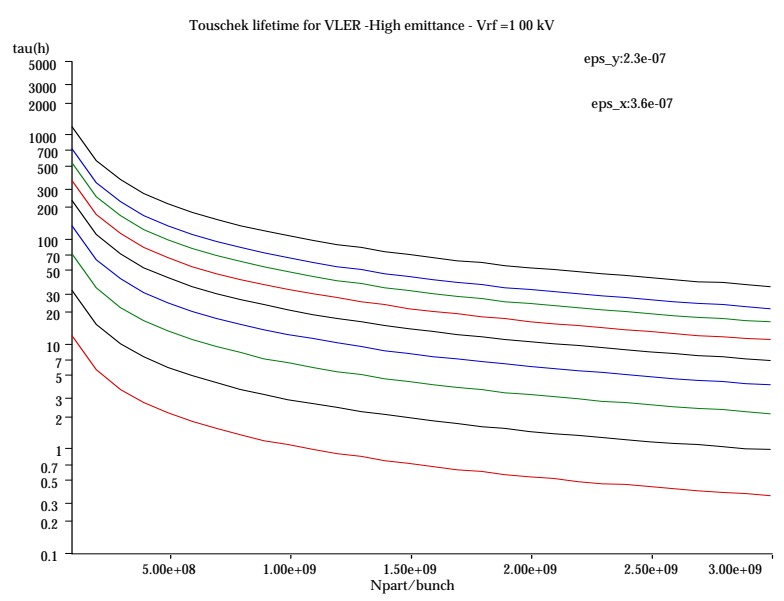

Figure 3:Touschek Lifetime at different beam energies.

Each curve refers to an energy value, ranging from 100 $\mathrm{MeV}$ (the lowest) up to $800 \mathrm{MeV}$ in $100 \mathrm{MeV}$ steps. Given the design charge per bunch at each energy, the shortest beam lifetime is larger than 2 hours for $100 \mathrm{MeV}$.
Of course a tracking of Touschek lost particles, taking into account the dynamic aperure and the real lattice at each energy will be performed. However even with a 30 min lifetime at the lowest operating energy, a gain in integrated luminosity could come from the possibility of inject in less than 5 minutes with the detector on, if the injection background rate can be kept low.

\subsection{Beam-Beam}

The large asymmetry of the two rings, and the fact that each bunch in VLER collides with many LER bunches can lead to instabilities due to barycentre coherent motion (dipole) [8]. We believe that a strong transverse bunchby-bunch feedback (as in PEP-II) can help. Very small (0.004) tune shifts in the LER and a careful choice of tunes will also help. We also know from PEP-II experience that the beam-beam force is a strong damper. A beam-beam simulation is needed to check this extreme weak-strong case.

\section{OPEN ISSUES}

The VLER design presents some special features that will require dedicated studies:

- The lattice design at low (100 MeV) and high (800 $\mathrm{MeV}$ ) energies, with constant emittance.

- Chromaticity correction and dynamic aperture.

- The Touschek lifetime can be a problem below $150 \mathrm{MeV}$. Possible solutions: longer bunch length, larger RF momentum acceptance, fast and efficient injection.

- The long damping times at lower energies. The segmented dipoles and a wiggler will help.

- The beam-beam case for asymmetric colliding bunches.

\section{CONCLUSIONS}

The PEP-N project is in its design phase. A 'proof-ofprinciple' design of the small ring for $500 \mathrm{MeV}$ has been given. The work on a high $(800 \mathrm{MeV})$ and low (300 $\mathrm{MeV}$ ) lattice is in progress and looks promising. A Workshop on the physics case was held at SLAC on April 30/May 1-2, 2001. A more detailed project design will be presented for discussion at SLAC in September 2001.

\section{REFERENCES}

[1] J. Seeman et al, 'PEP-N: a $0.8 \mathrm{GeVx} 3.1 \mathrm{GeV}$ e+e- collider at SLAC',RPPH138, this Conference.

[2] M. Sullivan et al, 'The PEP-N Interaction Region', RPPH139, this Conference.

[3] PEP-N Project LOI, PEP-II AP Note 2000.05, Sep. 2000, http://pep-n.pd.infn.it/LoI/loi_accelerator.html

[4] See Physics LoI in http://pep-n.pd.infn.it/

[5] A. Piwinsky,'Intrabeam scattering'. Proc.CAS CERN 87-03.

[6] H. Grote, F. Ch. Iselin, CERN/SI/90-13(AP).

[7] J. Le Duff, 'Single and multiple Touschek effect', Proc.CAS CERN 89-01.

[8] K. Hirata, E. Keil,, NIM A292 (1990). 